\title{
150.
}

\section{A MEMOIR ON THE CONDITIONS FOR THE EXISTENCE OF GIVEN SYSTEMS OF EQUALITIES AMONG THE ROOTS OF AN EQUATION.}

[From the Philosophical Transactions of the Royal Society of London, vol. CxLvII. for the year 1857, pp. 727-731. Received December 18, 1856,-Read January 8, 1857.]

IT is well known that there is a symmetric function of the roots of an equation, viz. the product of the squares of the differences of the roots, which vanishes when any two roots are put equal to each other, and that consequently such function expressed in terms of the coefficients and equated to zero, gives the condition for the existence of a pair of equal roots. And it was remarked long ago by Professor Sylvester, in some of his earlier papers in the Philosophical Magazine, that the like method could be applied to finding the conditions for the existence of other systems of equalities among the roots, viz. that it was possible to form symmetric functions, each of them a sum of terms containing the product of a certain number of the differences of the roots, and such that the entire function might vanish for the particular system of equalities in question; and that such functions expressed in terms of the coefficients and equated to zero would give the required conditions. The object of the present memoir is to extend this theory and render it exhaustive, by showing how to form a series of types of all the different functions which vanish for one or more systems of equalities among the roots; and in particular to obtain by the method distinctive conditions for all the different systems of equalities between the roots of a quartic or a quintic equation, viz. for each system conditions which are satisfied for the particular system, and are not satisfied for any other systems, except, of course, the more special systems included in the particular system. The question of finding the conditions for any particular system of equalities is essentially an indeterminate one, for given any set of functions which vanish, a function syzygetically connected with these will also vanish; the discussion of the nature of the

c. II. 
syzygetic relations between the different functions which vanish for any particular system of equalities, and of the order of the system composed of the several conditions for the particular system of equalities, does not enter into the plan of the present memoir. I have referred here to the indeterminateness of the question for the sake of the remark that I have availed myself thereof, to express by means of invariants or covariants the different systems of conditions obtained in the sequel of the memoir; the expressions of the different invariants and covariants referred to are given in my 'Second Memoir upon Quantics,' Philosophical Transactions, vol. CXLVI. (1856), [141].

1. Suppose, to fix the ideas, that the equation is one of the fifth order, and call the roots $\alpha, \beta, \gamma, \delta, \epsilon$. Write $12=\Sigma \phi(\alpha-\beta)^{l}, \quad 12.13=\Sigma \phi(\alpha-\beta)^{l}(\alpha-\gamma)^{m}, \quad 12.34=$ $\Sigma \phi(\alpha-\beta)^{l}(\gamma-\delta)^{n}$, \&c., where $\phi$ is an arbitrary function and $l, m$, \&c. are positive integers. It is hardly necessary to remark that similar types, such as $12,13,45$, \&c., or as 12.13 and 23.25, \&c., denote identically the same sums. Two types, such as 12.13 and 14.15.23.24.25.34.35.45, may be said to be complementary to each other. A particular product $(\alpha-\beta)(\gamma-\delta)$ does or does not enter as a term (or factor of a term) in one of the above-mentioned sums, according as the type 12.34 of the product, or some similar type, does or does not form part of the type of the sum; for instance, the product $(\alpha-\beta)(\gamma-\delta)$ is a term (or factor of a term) of each of the sums 12.34, 13.45.24, \&c., but not of the sums 12.13.14.15, \&c.

2. If, now, we establish any equalities between the roots, e.g. $\alpha=\beta, \gamma=\delta$, the effect will be to reduce certain of the sums to zero, and it is easy to find in what cases this happens. The sum will vanish if each term contains one or both of the factors $\alpha-\beta, \gamma-\delta$, i.e. if there is no term the complementary of which contains the product $(\alpha-\beta)(\gamma-\delta)$, or what is the same thing, whenever the complementary type does not contain as part of it, a type such as 12.34. Thus for the sum 14.15.24.25.34.35.45, the complementary type is 12.13 .23 , which does not contain any type such as 12.34, i. e. the sum 14.15.24.25.34.35.45 vanishes for $\alpha=\beta, \gamma=\delta$. It is of course clear that it also vanishes for $\alpha=\beta=\epsilon, \gamma=\delta$ or $\alpha=\beta=\gamma=\delta$, \&c., which are included in $\alpha=\beta, \gamma=\delta$. But the like reasoning shows, and it is important to notice, that the sum in question does not vanish for $\alpha=\beta=\gamma$ : and of course it does not vanish for $\alpha=\beta$. Hence the vanishing of the sum 14.15.24.25.34.35.45 is characteristic of the system $\alpha=\beta, \gamma=\delta$. A system of roots $\alpha, \beta, \gamma, \delta, \in$ may be denoted by 11111 ; but if $\alpha=\beta$, then the system may be denoted by 2111, or if $\alpha=\beta, \gamma=\delta$, by 221 , and so on. We may then say that the sum 14.15.24.25.34.35.45 does not vanish for 2111 , vanishes for 221 , does not vanish for 311 , vanishes for $32,41,5$.

3. For the purpose of obtaining the entire system of results it is only necessary to form Tables, such as the annexed Tables, the meaning of which is sufficiently explained by what precedes: the mark $(x)$ set against a type denotes that the sum represented by the complementary type vanishes, the mark ( $(0)$ that the complementary type does not vanish, for the system of roots denoted by the symbol at the top or bottom of the column; the complementary type is given in the same horizontal line with the original type. It will be noticed that the right-hand columns do not extend to the foot of the Table; the reason of this of course is, to avoid a repetition of the same type. Some of 
the types at the foot of the Tables are complementary to themselves, but I have, notwithstanding this, given the complementary type in the form under which it naturally presents itself.

4. The Tables are:

Table for the equal Roots of a Quartic.

\begin{tabular}{|c|c|c|c|c|c|c|c|c|c|c|}
\hline 211 & 22 & 31 & 4 & & & & $211 \mid$ & 22 & 31 & 4 \\
\hline$x$ & $x$ & $x$ & $x$ & 12.13 . & 14 & $23 \cdot 24 \cdot 34$ & 0 & 0 & o & 0 \\
\hline 0 & $x$ & $x$ & $x$ & $\overline{12} 13$. & 14. & $23 \cdot 24 \cdot 34$ & o & o & o & $x$ \\
\hline o & $x$ & $x$ & $x$ & $12 \div 13$ & $14:$ & $23 \cdot 24 \cdot 34$ & o & o & o & $\times$ \\
\hline o & o & $x$ & $x$ & 12.34 & 13. & $14 \cdot 23 \cdot 24$ & o & 0 & $x$ & $x$ \\
\hline o & $x$ & $x$ & $\times$ & 12.13. & 14 & $23 \cdot 24 \cdot 34$ & & & & \\
\hline o & o & $x$ & $\times$ & 12.13 . & 24 & $14.23 \ldots 34$ & 211 & 22 & 31 & 4 \\
\hline o & $x$ & 0 & $\times$ & 12.13 . & 23 & $14 \cdot 24 \cdot 34$ & & & & \\
\hline 11 & 22 & 31 & 4 & & & & & & & \\
\hline
\end{tabular}

Table for the equal Roots of a Quintic.

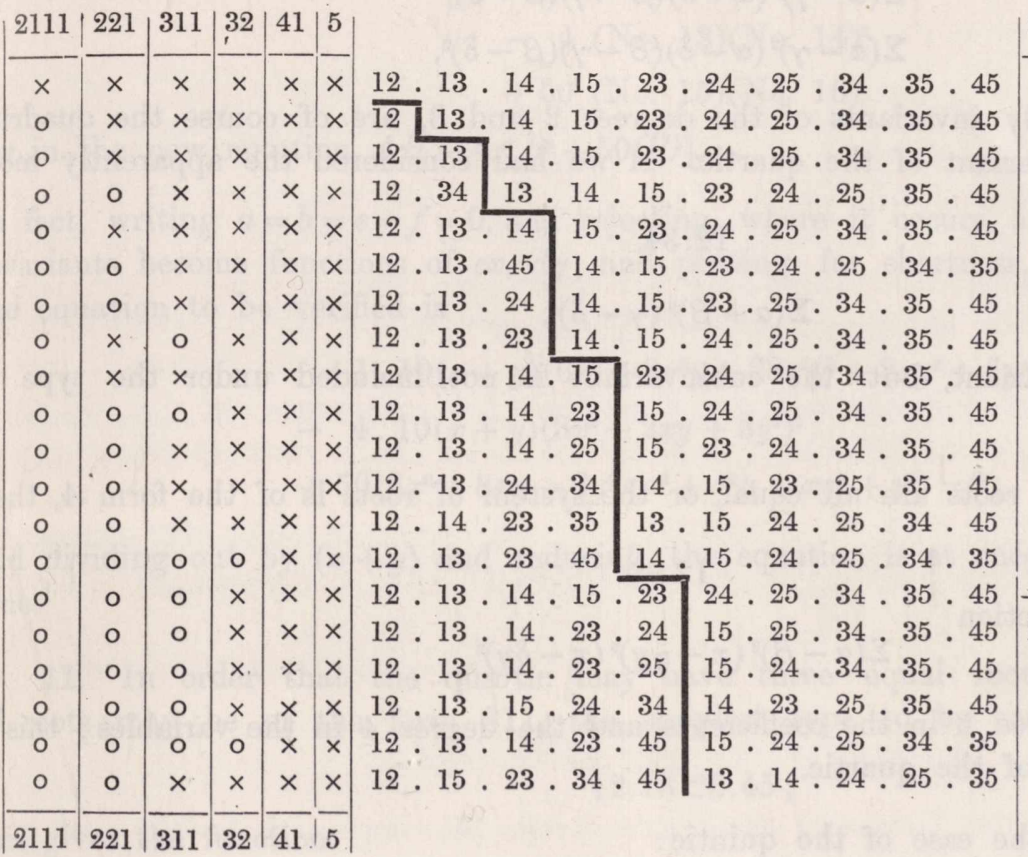

\begin{tabular}{|c|c|c|c|c|c|}
2111 & 221 & 311 & 32 & 41 & 5 \\
\hline 0 & 0 & 0 & 0 & 0 & 0 \\
0 & 0 & 0 & 0 & 0 & $x$ \\
0 & 0 & 0 & 0 & 0 & $\times$ \\
0 & 0 & 0 & 0 & $\times$ & $\times$ \\
0 & 0 & 0 & 0 & 0 & $\times$ \\
0 & 0 & 0 & 0 & $\times$ & $\times$ \\
0 & 0 & 0 & 0 & $\times$ & $\times$ \\
0 & 0 & 0 & $\times$ & $\times$ & $\times$ \\
0 & 0 & 0 & $\times$ & 0 & $\times$ \\
0 & 0 & 0 & $\times$ & $\times$ & $\times$ \\
0 & 0 & 0 & $\times$ & $\times$ & $\times$ \\
0 & 0 & 0 & 0 & $\times$ & $\times$ \\
0 & 0 & 0 & 0 & $\times$ & $\times$ \\
0 & 0 & $\times$ & $\times$ & $\times$ & $\times$ \\
\hline 2111 & 221 & 311 & 32 & 41 & 5
\end{tabular}

The two Tables enable the discussion of the theory of the equal roots of a quartic or quintic equation: first for the quartic:

5. In order that a quartic may have a pair of equal roots, or what is the same thing, that the system of roots may be of the form 211 , the type to be considered is 
this of course gives as the function to be equated to zero, the discriminant of the quartic.

6. In order that there may be two pairs of equal roots, or that the system may be of the form 22 , the simplest type to be considered is

this gives the function

$$
\text { 14.24.34; }
$$

$$
\Sigma(\alpha-\delta)(\beta-\delta)(\gamma-\delta)(x-\alpha y)^{2}(x-\beta y)^{2}(x-\gamma y)^{2},
$$

which being a covariant of the degree 3 in the coefficients and the degree 6 in the variables, can only be the cubicovariant of the quartic.

7. In order that the quartic may have three equal roots, or that the system of roots may be of the form 31 , we may consider the type

13.14.23.24,

and we obtain thence the two functions

$$
\begin{aligned}
& \Sigma(\alpha-\gamma)(\alpha-\delta)(\beta-\gamma)(\beta-\delta), \\
& \Sigma(\alpha-\gamma)^{2}(\alpha-\delta)(\beta-\gamma)(\beta-\delta)^{2},
\end{aligned}
$$

which being respectively invariants of the degrees 2 and 3 , are of course the quadrinvariant and the cubinvariant of the quartic. If we had considered the apparently more simple type

this gives the function

$$
12.34 \text {, }
$$

$$
\Sigma(\alpha-\beta)^{3}(\gamma-\delta)^{2},
$$

which is the quadrivariant, but the cubinvariant is not included under the type in question.

8. Finally, if the roots are all equal, or the system of roots is of the form 4, then the simplest type is

and this gives the function

12 ;

$$
\Sigma(\alpha-\beta)^{2}(x-\gamma y)^{2}(x-\delta y)^{2},
$$

a covariant of the degree 2 in the coefficients and the degree 4 in the variables; this is of course the Hessian of the quartic.

Considering next the case of the quintic:

9. In order that a quintic may have a pair of equal roots, or what is the same thing, that the system of roots may be of the form 2111, the type to be considered is

$$
\text { 12.13.14.15.23.24.25.34.35.45; }
$$

this of course gives as the function to be equated to zero, the discriminant of the quintic. 
10. In order that the quintic may have two pairs of equal roots, or that the system of roots may be 221 , the simplest type to be considered is

$$
14.15 .24 .25 .34 .35 .45 \text {; }
$$

a type which gives the function

$$
\Sigma(\alpha-\delta)(\alpha-\epsilon)(\beta-\delta)(\beta-\epsilon)(\gamma-\delta)(\gamma-\epsilon)(\delta-\epsilon)^{2}(x-\alpha y)^{3}(x-\beta y)^{3}(x-\gamma y)^{3} .
$$

This is a covariant of the degree 5 in the coefficients and of the degree 9 in the variables; but it appears from the memoir above referred to, that there is not any irreducible covariant of the form in question; such covariant must be a sum of the products (No. 13)(No. 20), (No. 13)(No. 14)2, (No. 15)(No. 16) (the numbers refer to the Covariant Tables given in the memoir), each multiplied by a merely numerical coefficient. These numerical coefficients may be determined by the consideration that there being two pairs of equal roots, we may by a linear transformation make these roots $0,0, \infty, \infty$, or what is the same thing, we may write $a=b=e=f=0$, the covariant must then vanish identically. The coefficients are thus found to be $1,-4,50$, and we have for a covariant vanishing in the case of two pairs of equal roots,

$$
\begin{array}{r}
1(\text { No. } 13)(\text { No. } 20) \\
-4(\text { No. } 13)(\text { No. } 14)^{2} \\
+50(\text { No. } 15)(\text { No. } 16)
\end{array}
$$

[or in the new notation $A H-4 A B^{2}+50 C D$ ].

In fact, writing $a=b=e=f=0$, and rejecting, where it occurs, a factor $x^{2} y^{3}$, the several covariants become functions of $c x, d y$; and putting, for shortness, $x, y$ instead of $c x, d y$, the equation to be verified is

$$
\begin{aligned}
& 1.10(x+y)\left(6 x^{4}+8 x^{3} y+28 x^{2} y^{2}+8 x y^{3}+6 y^{4}\right) \\
- & 4.10(x+y)\left(3 x^{2}+2 x y+3 y^{2}\right)^{2} \\
+ & 50\left(6 x^{2}+8 x y+6 y^{2}\right)\left(x^{3}+x^{2} y+x y^{2}+y^{3}\right)=0
\end{aligned}
$$

and dividing out by $(x+y)$ and reducing, the equation is at once seen to be identically true.

11. In order that the quintic may have three equal roots, or that the system of roots may be of the form 311, the simplest type to be considered is

$$
\text { 12.13.23.45; }
$$

this gives the function

$$
\Sigma(\alpha-\beta)^{2}(\beta-\gamma)^{2}(\gamma-\alpha)^{2}(\delta-\epsilon)^{4},
$$

which being an invariant, and being of the fourth degree in the coefficients, must be the quartinvariant of the quintic [that is No. 19, =G]. The same type gives also the function

$$
\Sigma(\alpha-\beta)^{2}(\beta-\gamma)^{2}(\gamma-\alpha)^{2}(\delta-\epsilon)^{2}(x-\delta y)^{2}(x-\epsilon y)^{2},
$$


which is a covariant of the degree 4 in the coefficients and the degree 4 in the variables; and it must vanish when $a=b=c=0$, this can only be the covariant

$$
3(\text { No. } 20)-2(\text { No. } 14)^{2},\left[=3 H-2 B^{2}\right],
$$

which it is clear vanishes as required.

12. In order that the quintic may have three equal roots and two equal roots, or that the system of roots may be of the form 32 , the simplest type to be considered is

which gives the function

$$
\text { 12.13.14.15, }
$$

$$
\Sigma(\alpha-\beta)(\alpha-\gamma)(\alpha-\delta)(\alpha-\epsilon)(x-\beta y)^{3}(x-\gamma y)^{3}(x-\delta y)^{3}(x-\epsilon y)^{3},
$$

a covariant of the degree 4 in the coefficients, and the degree 12 in the variables; and it must vanish when $a=b=c=0, e=f=0$; this can only be the covariant

$$
3(\text { No. } 13)^{2}(\text { No. } 14)-25(\text { No. } 15)^{2},\left[=3 A^{2} B-25 C^{2}\right] \text {, }
$$

which it is clear vanishes as required.

13. In order that the quintic may have four equal roots, or that the system may be of the form 41, the simplest type to be considered is

$$
12.34 \text {, }
$$

which gives the function

$$
\Sigma(\alpha-\beta)^{2}(\gamma-\delta)^{2}(x-\epsilon y)^{2},
$$

a covariant of the degree 2 in the coefficients, and of the same degree in the variables; this can only be the covariant (No. 14), [=B].

14. Finally, in order that all the roots may be equal, or that the system of roots may be of the form 5 , the type to be considered is

and this gives the function

$$
12 \text {; }
$$

$$
\Sigma(\alpha-\beta)^{2}(x-\gamma y)^{2}(x-\delta y)^{2}(x-\epsilon y)^{2},
$$

a covariant of the degree 2 in the coefficients, and the degree 6 in the variables, and this can only be the Hessian (No. 15), $[=C]$.

It will be observed that all the preceding conditions are distinctive; for instance, the covariant which vanishes when the system of roots is of the form 311, does not vanish when the system is of the form-221, or of any other form not included in the form 311 . 